\title{
Preparation of Janus Fabric by PVDF Electrospinning Technology and Its Unidirectional Water/Moisture Transportation Performance
}

\author{
Chuntao Wu1, Omar Faruk ${ }^{2}$, Qianhong Jin ${ }^{3}$, Guobao Wang ${ }^{3}$, Jindan $\mathrm{Wu}^{2}$, Dongming $\mathrm{Qi}^{2 *}$ \\ ${ }^{1}$ Ningbo Vehicle Emission Pollution Control and Administration Center, Ningbo, China \\ ${ }^{2}$ College of Textile Science and Engineering, Zhejiang Sci-Tech University, Hangzhou, China \\ ${ }^{3}$ Yiwu Zhongli Industry and Trade Co. Ltd., Yiwu, China \\ Email: *dongmingqi@zstu.edu.cn
}

How to cite this paper: Wu, C.T., Faruk, O., Jin, Q.H., Wang, G.B., Wu, J.D. and Qi, D.M. (2020) Preparation of Janus Fabric by PVDF Electrospinning Technology and Its Unidirectional Water/Moisture Transportation Performance. Journal of Textile Science and Technology, 6, 144-152.

https://doi.org/10.4236/jtst.2020.63012

Received: July 25, 2020

Accepted: August 18, 2020

Published: August 21, 2020

Copyright $\odot 2020$ by author(s) and Scientific Research Publishing Inc. This work is licensed under the Creative Commons Attribution International License (CC BY 4.0).

http://creativecommons.org/licenses/by/4.0/

\begin{abstract}
Directional fluid transport is of significance to many physical processes in nature. How to manipulate this process by man-made material is still a key challenge to scientists. In this study, Janus fabric was constructed by electrospinning a layer of polyvinylidene fluoride (PVDF) nanofibers on woven cotton or gauze. The chemical composition, morphology and surface wettability of two sides of Janus fabric were characterized by infrared spectroscopy, scanning electron microscope (SEM) and contact angle measurement. By controlling the PVDF electrospinning time, the maximum hydrostatic pressure of Janus fabric with different PVDF thickness was measured. It was found that PVDF/gauze is more favorable for unidirectional water transportation, and the moisture also can transfer from hydrophobic side to hydrophilic side. With the advantages of facile preparation, low-cost and one-way water/moisture transportation, the Janus fabric prepared in this study can be applied for water separation, humidity transfer and water collection from the air.
\end{abstract}

\section{Keywords}

Unidirectional Water Transportation, Surface Wettability, Nanofiber, Hydrostatic Pressure

\section{Introduction}

Liquid unidirectional transportation refers to the phenomenon that the liquid 
spontaneously penetrates along a certain direction without the external energy input (such as pressure field, light, magnetism, etc.), but does not transport in the opposite direction [1] [2] [3]. This phenomenon can be observed in the nature, such as the water collecting function of spider silk [4], the unidirectional flow of cell membrane liquid, etc. The self-transportation of water or moisture could be applied in the waterproof and moisture permeable clothing [5] [6], water collection [7] [8], water separation and purification, etc.

Janus membrane, with opposite wettability in the both sides, is found to be effective in driving liquid unidirectional transportation [9] [10] [11]. However, how to introduce surfaces with different wettability to the porous membrane, is a great challenge to the scientists [12] [13]. For wet modification method, the modification of one side is hard to realize due to the capillary force existing in porous matrix. In recent years, electrospinning, as an efficient and convenient method for the preparation of nanofibers, has been used to prepare Janus membrane [14] [15] [16]. Electrospinning is a method that uses high-voltage electrostatic force to stretch and refine polymer solution or melt, and finally solidify on the receiving substrate to prepare micron or nanofibers [17].

It is reported that the structure of electrospun fibers is affected by many factors, including the type and the concentration of polymer solution, electrospinning voltage, receiving distance, the humidity and so on [18]. For example, by controlling the concentration of spinning solution, the "bead-knot" structure fiber can be prepared to enhance the hydrophobicity of the material and achieve a stable superhydrophobic state [19]. Moreover, the thickness and porosity of nanofibrous membrane could be regulated by adjusting the electrospinning time or receiving distance. Therefore, by using electrospinning technology, the composition and structure of Janus membrane could be well controlled [20] [21].

Janus membrane can effectively drive the self-transportation of liquid. When the water droplet drops on the hydrophobic side, the contact angle is larger than $90^{\circ}$ due to the hydrophobic effect and the surface tension of water itself. Under the co-interaction of Laplace pressure and capillary force, the water droplet is quickly pulled into the hydrophilic side to achieve the spontaneous transport through the membrane [22]. The inverse process would be blocked since the water droplet will spread on the surface as soon as it contacts the hydrophilic side. In this study, a Janus membrane was prepared by electrospinning poly (vinylidene fluoride) (PVDF) nanofibers onto cotton fabric (woven cotton and gauze). The thickness of PVDF layer was regulated and the unidirectional water transportation performance was investigated.

\section{Experimental Section}

\subsection{Materials}

PVDF (180,000 MW, Sigma-Aldrich) and N,N-Dimethylacetamide (DMF, 99.5\%, Gaojing Fine Chemical Industry Co., Ltd.) were used as received. Medical gauze and woven cotton fabric were purchased from Jingzhong Sanitary Mate- 
rials Co. Ltd. and Hengyang Textile Co. Ltd., respectively.

\subsection{Preparation of PVDF/Cotton Fabric}

$2.5 \mathrm{~g}$ PVDF and $7.5 \mathrm{~g}$ DMF were added into a glass vial, followed by being stirred at $75^{\circ} \mathrm{C}$. The obtained $25 \mathrm{wt} \%$ PVDF solution was used for electrospinning. The syringe was fixed on the propelling pump. The high-voltage positive stage was connected to the spinneret, and the negative electrode was connected to the receiving device. The fabric $(20 \mathrm{~cm} \times 30 \mathrm{~cm})$ was fixed on the aluminum foil and used as a receiving substrate. The receiving distance was $15 \mathrm{~cm}$ and the voltage was set at $16 \mathrm{KV}$. The electrospinning speed was $0.15 \mathrm{~mm} / \mathrm{min}$, and the electrospinning time was $2,4,6,8,10,15,20,25,30 \mathrm{~min}$.

\subsection{Characterization}

The prepared Janus films were characterized by Nicolet 5700 Fourier transform infrared spectroscopy (FTIR). The chemical components of two sides of Janus fabric were analyzed.

Scanning electron microscope (JSM-5610LV) was used to observe the morphology of different electrospun membranes. SEM uses electron beams to scan the sample surface point by point, and the generated electronic signals display various characteristics of the surface morphology. By adjusting the electrospinning time, the structure of different PVDF membranes was investigated.

The wettability of both sides of Janus fabric was measured by DSA-20 video contact angle tester. The water droplet $(2 \mu \mathrm{L})$ was dropped onto the fabric and the variation of contact angle was recorded and the wicking curve was analyzed.

\subsection{Hydrostatic Test}

The hydrostatic test was carried out by putting the Janus fabric between two filter cups, and water was pumped into the upper cup by peristaltic pump at a flow rate of $39 \mathrm{~mL} / \mathrm{min}$. The height of water when it begins to penetrate was recorded, and the maximum hydrostatic pressures that both sides of Janus fabric can hold were studied.

\subsection{Moisture Management test}

The sample $(90 \mathrm{~mm} \times 90 \mathrm{~mm})$ was put on the sensor of the instrument. The water dripping time was set as $10 \mathrm{~s}$. The moisture content of the upper and lower side of fabric was measured and the total test time was $260 \mathrm{~s}$. And then, the accumulating one-way transport index was calculated by the instrument.

\section{Results and Discussion}

Two types of fabrics were used as substrates to prepare Janus fabric by introducing PVDF nanofibers on their upper surfaces. PVDF was served as hydrophobic layer and the cotton fabrics were served as hydrophilic layer. The surface chemistry and wettability were investigated to confirm the success of preparing Janus fabric. 


\subsection{Surface Chemistry of Janus Fabric}

FTIR test was employed to analyze the different chemical composition of the hydrophilic and hydrophobic sides of Janus fabric (Figure 1). It was shown that new peaks were found in the spectra of hydrophobic side of Janus fabric. The peak at $1166 \mathrm{~cm}^{-1}$ was attributed to the stretching vibration of $-\mathrm{CF}_{2}$, which is a side group of PVDF chain. For the hydrophilic side, peaks at $1108 \mathrm{~cm}^{-1}$ and 1056 $\mathrm{cm}^{-1}$ is the main characteristic peaks of cotton. It was indicated that the chemical composition of two sides is different and the Janus fabric has been successfully prepared.

\subsection{Surface Morphology of Janus Fabric}

The structures of PVDF nanofibers on woven cotton and gauze were observed by SEM (Figure 2). The as-prepared PVDF nanofibers have spindle structures in the filaments, which may beneficial to the improvement of surface hydrophobicity. According to the Wenzel model, the increase of surface roughness by introducing microstructure into the nanofibers will lead to the enhancement of surface hydrophobicity.

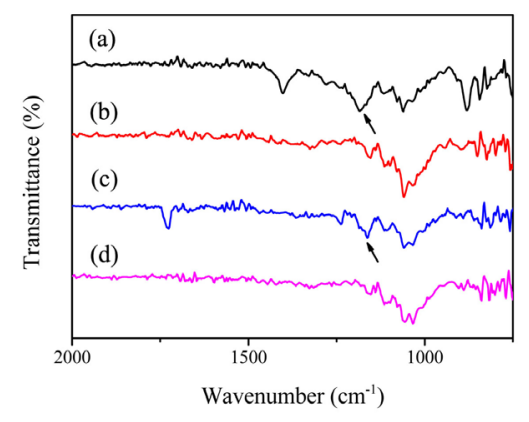

Figure 1. FTIR spectra of Janus fabrics: (a) hydrophobic side of PVDF/woven cotton; (b) hydrophilic side of PVDF/woven cotton; (c) hydrophobic side of PVDF/gauze; (d) hydrophilic side of PVDF/gauze.

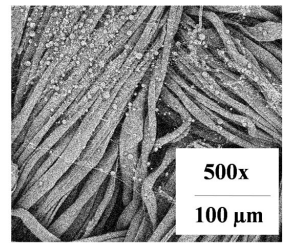

(a)

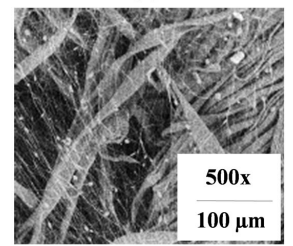

(e)

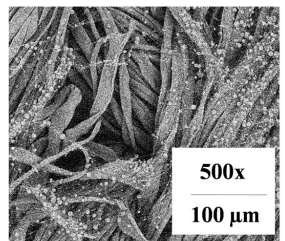

(b)

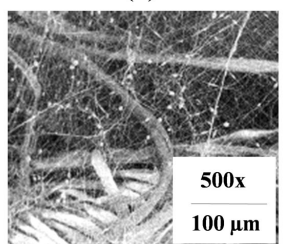

(f)

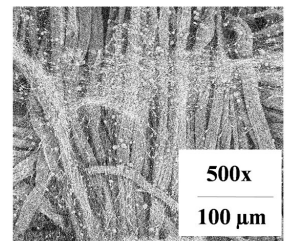

(c)

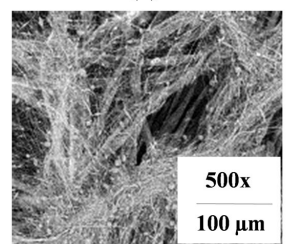

(g)

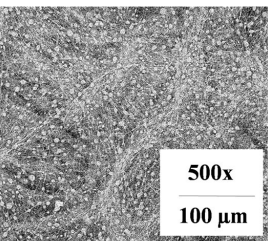

(d)

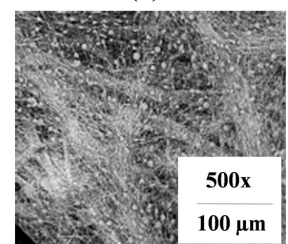

(h)

Figure 2. SEM images of PVDF nanofibers on woven cotton and gauze. ((a)-(d)) PVDF nanofibers on woven cotton: (a) $6 \mathrm{~min}$; (b) $8 \mathrm{~min}$; (c) $10 \mathrm{~min}$ and (d) $20 \mathrm{~min}$; ((e)-(h)) PVDF nanofibers on gauze: (e) $2 \mathrm{~min}$; (f) $4 \mathrm{~min}$; (g) $6 \mathrm{~min}$ and (h) $10 \mathrm{~min}$. 
Not only the hydrophobicity, but also the thickness of hydrophobic layer has a significant influence on the unidirectional water transportation performance. The thickness of PVDF nanofiber membrane was controlled by changing the electrospinning time. As shown in Figure 2, with the increase of PVDF electrospinning time, nanofibers with a higher density were found on the surface of cotton fabric. When the electrospinning time is short (e.g. $6 \mathrm{~min}$ and $8 \mathrm{~min}$ ), the PVDF nanofiber layer is too thin to cover the pores of cotton fabric. When the electrospinning time is 10 min or longer, a layer of uniformly distributed nanofibers was formed on both woven cotton and gauze (Figure 2(c), Figure 2(d), Figure 2(h)).

\subsection{Surface Wettability of Janus Fabric}

The surface wettability of Janus fabric was characterized by contact angle (CA) measurement (Figure 3$)$. The cotton fabric is hydrophilic with $\mathrm{CA}=0^{\circ}(\mathrm{t}=0 \mathrm{~s})$, while the PVDF membrane is hydrophobic with CA approximately $130^{\circ}(\mathrm{t}=0$ s). For PVDF/woven cotton, the PVDF layer is still hydrophilic when the electrospinning time is $2 \mathrm{~min}$ or $4 \mathrm{~min}$ (Figure $3(\mathrm{a})$ ). It is because that PVDF nanofibers is too thin to cover the hydrophilic substrate completely. As the thickness of PVDF layer further increased (the electrospinning time $>4 \mathrm{~min}$ ), insignificant change of contact angle was found. For PVDF/gauze, the variation of contact angle showed a similar trend.

Since cotton fabric is hydrophilic, water droplet will easily wick into the fabric due to the capillary effect. The wetting behavior of water droplet on the surface of PVDF/woven cotton and PVDF-gauze was investigated. When the electrospinning time is $4 \mathrm{~min}$ and $6 \mathrm{~min}$, the PVDF nanofibers were completely wetted within $3 \mathrm{~s}$ (data is not shown here). When the electrospinning time is $8 \mathrm{~min}$, the water contact angle of PVDF layer slightly reduced about $5^{\circ}$ at $t=5 \mathrm{~min}$ (Figure 3(b)). For PVDF/woven cotton with PVDF electrospinning time $10 \mathrm{~min}$, the water droplet was very stable. There is no obvious change was found in CA value.

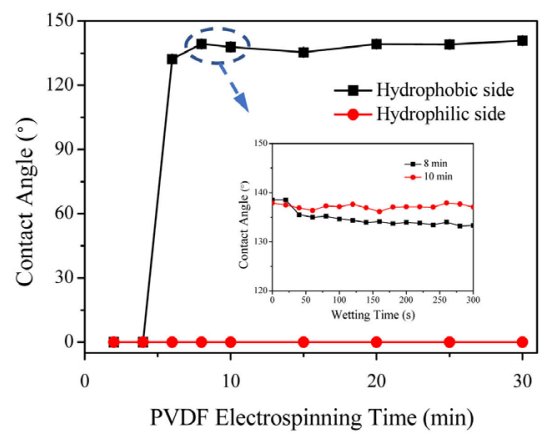

(a)

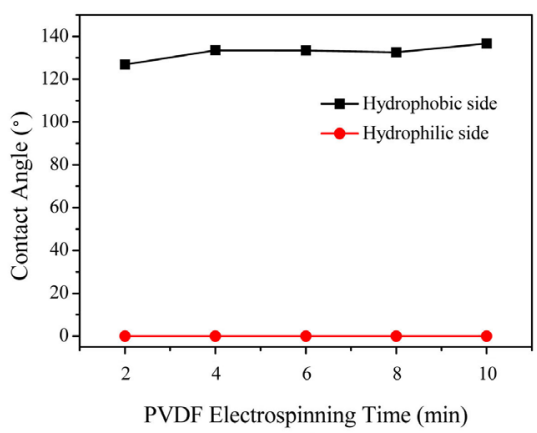

(b)

Figure 3. Water contact angle $(t=0 \mathrm{~s})$ of Janus fabric. (a) The contact angle of $\mathrm{PVDF} /$ woven cotton. The inset is the wick curve of PVDF layer of PVDF/woven cotton with PVDF electrospinning time $8 \mathrm{~min}$ and $10 \mathrm{~min}$. (b) The contact angle of PVDF/gauze. 
The images of water droplets on hydrophobic side and hydrophilic side of Janus fabric are consistent with the above results (Figure 4). Due to the existence of PVDF nanofibrous membrane on the hydrophobic side, the water droplet cannot wick into the fabric, while it rapidly goes into the fabric on the hydrophilic side. Therefore, it can be concluded that we have successfully prepared a Janus fabric with opposite wettability on two sides.

\subsection{Unidirectional Water Transportation Performance}

When the electrospinning time was $2 \mathrm{~min}$ and $4 \mathrm{~min}$, the maximum hydrostatic pressure of the hydrophilic side was lower than that of the hydrophobic side (Figure 5(a)). It is because PVDF nanofibers are not thick enough to resist the penetrating of water. When the electrospinning time was $6 \mathrm{~min}$ and $8 \mathrm{~min}$, the maximum hydrostatic pressure of the hydrophilic side was lower than that of the hydrophobic side. When the electrospinning time is $10 \mathrm{~min}$, water penetration can be blocked in a certain level by the dense and thick PVDF nanofibrous layer on the fabric. In this situation, it is easier for water to transport from hydrophilic side to hydrophobic side. Therefore, for PVDF/woven cotton, water is failed to transport unidirectionally.

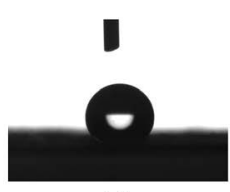

(a)

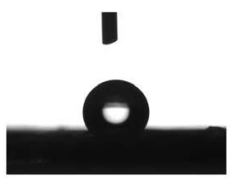

(e)

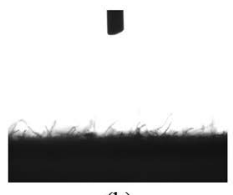

(b)

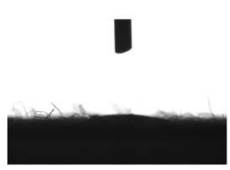

(f)

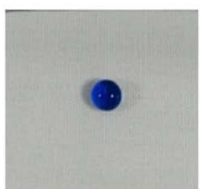

(c)

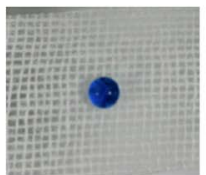

(g)

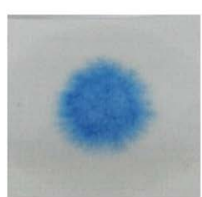

(d)

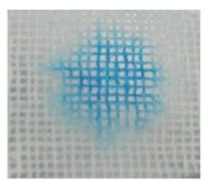

(h)

Figure 4. The images of water droplets on ((a), (e)) hydrophobic side and ((b), (f)) hydrophilic side of PVDF/woven cotton with PVDF electrospinning time $8 \mathrm{~min}$; The images of water droplet on ((c), (g)) hydrophobic side and ((d), (h)) hydrophilic side of PVDF/gauze with PVDF electrospinning time $6 \mathrm{~min}$.

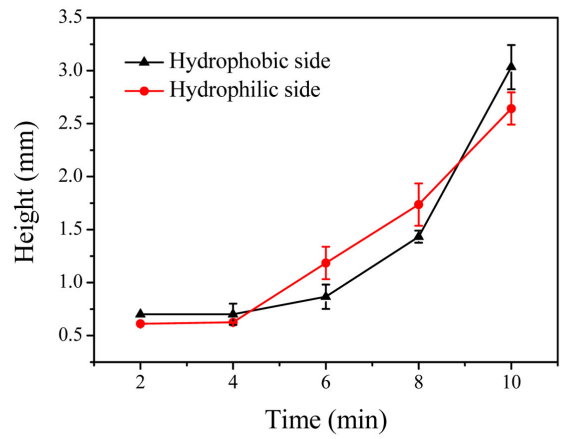

(a)

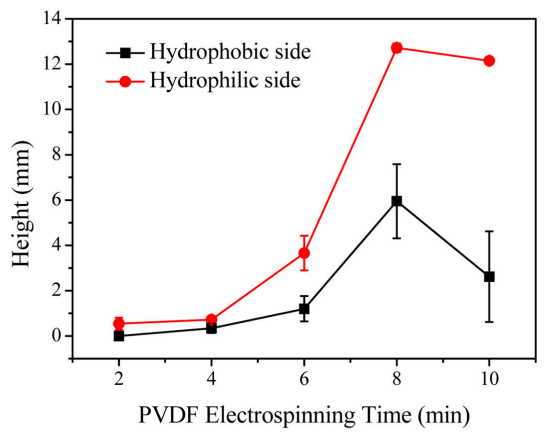

(b)

Figure 5. Hydrostatic pressure resistance test: (a) the height of water column on both sides of PVDF/woven cotton; (b) the height of water column on both sides of PVDF/gauze. 
For PVDF/gauze, the maximum hydrostatic pressure of hydrophilic side is always larger than that of hydrophobic side (Figure 5(b)). It suggested that water tend to transport from hydrophobic side to hydrophilic side. When the electrospinning time is $8 \mathrm{~min}$, the thickness of PVDF layer is optimal for driving the unidirectional water transportation since the difference of height between two side is the largest. In conclusion, $\mathrm{PVDF} /$ gauze is more conducive to the one-way water transport. It may be attributed to a relatively high porosity of gauze comparing to woven cotton. A larger capillary force makes it easier for water to overcome the hydrophobic force brought by PVDF layer and pass through from hydrophobic side to hydrophilic side.

The moisture permeability of Janus fabric was characterized by moisture management tester (MMT). This measurement is used to investigate the dynamic performance of liquid in fabrics, including the water absorption speed of inner and outer layers of fabric and one-way water transportation capability. As shown in Figure 6(a), the water ring area (indicated by blue) on the hydrophobic side of PVDF/gauze is smaller than that on the hydrophilic side. It is suggested that water unidirectionally transport from hydrophobic side to hydrophilic side. As a control, the water ring areas on both sides of gauze are similar, which indicated that water can reversely permeate from one side to the other (Figure 6(b)). The cumulative one-way transport index of the fabric is quantitatively analyzed (Figure 6(c)). The one-way transport index means the difference of liquid absorption and diffusion tendency from one side to the other. The results showed that the index of gauze is negative, while that of PVDF/gauze is 5.86. The positive value also proves that the water transportation in PVDF/gauze is unidirectional.

\section{Conclusion}

A layer of hydrophobic PVDF nanofibers was composited to hydrophilic cotton fabric by electrospinning technology. SEM images showed that the nanofibers owned spindle structure and the thickness and morphology of PVDF nanofibers

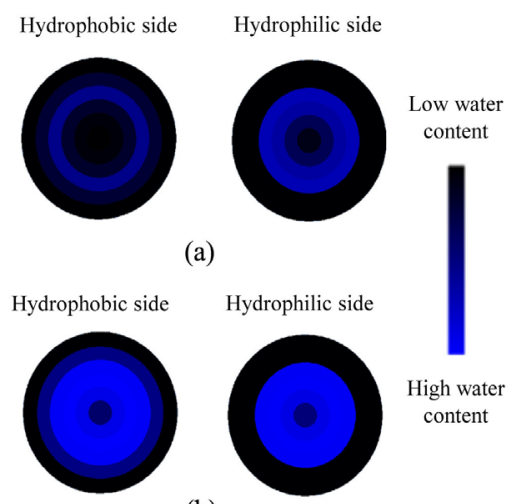

(b)

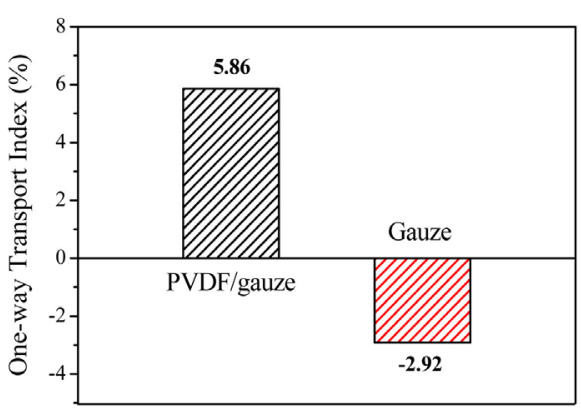

(c)

Figure 6. MMT test of (a) PVDF/gauze (PVDF electrospinning time is $8 \mathrm{~min}$ ) and (b) gauze; (c) one-way transport index of PVDF/gauze and gauze. 
were well controlled by adjusting the electrospinning time. The obtained $\mathrm{PVDF} /$ woven cotton and PVDF/gauze showed different chemical composition and wettability on two sides. The maximum hydrostatic pressure of hydrophilic side is larger than that of hydrophobic side of PVDF/gauze, indicating that it is easier for water transfer from hydrophobic side to hydrophilic side. The difference of hydrostatic pressure of two sides is prominently affected by the type of hydrophilic substrate and the thickness of hydrophobic layer. Our study provided a facile way to prepare Janus fabric with controllable structure and good unidirectional water transportation performance.

\section{Acknowledgements}

This work was supported by the Fundamental Research Funds of Zhejiang Sci-Tech University.

\section{Conflicts of Interest}

The authors declare no conflicts of interest regarding the publication of this paper.

\section{References}

[1] Chaudhury, M.K. and Whitesides, G.M. (1992) How to Make Water Run Uphill. Science, 256, 1539. https://doi.org/10.1126/science.256.5063.1539

[2] Hou, X. (2016) Smart Gating Multi-Scale Pore/Channel-Based Membranes. Advanced Materials, 28, 7049-7064. https://doi.org/10.1002/adma.201600797

[3] Sheng, Z.Z., Wang, H.L., Tang, Y.L., et al. (2018) Liquid Gating Elastomeric Porous System with Dynamically Controllable Gas/Liquid Transport. Science Advances, 4 , eaao6724. https://doi.org/10.1126/sciadv.aao6724

[4] Zheng, Y.M., Bai, H., Huang, Z., et al. (2010) Directional Water Collection on Wetted Spider Silk. Nature, 463, 640-643. https://doi.org/10.1038/nature08729

[5] Li, Y., Yang, F.F., Yu, J.Y., et al. (2016) Hydrophobic Fibrous Membranes with Tunable Porous Structure for Equilibrium of Breathable and Waterproof Performance. Advanced Materials Interfaces, 3, Article ID: 1600516. https://doi.org/10.1002/admi.201600516

[6] Li, Y., Zhu, Z.G., Yu, J.Y., et al. (2015) Carbon Nanotubes Enhanced Fluorinated Polyurethane Macroporous Membranes for Waterproof and Breathable Application. ACS Applied Materials \& Interfaces, 7, 13538-13546. https://doi.org/10.1021/acsami.5b02848

[7] Chen, W. and Guo, Z. (2019) Hierarchical Fibers for Water Collection Inspired by Spider Silk. Nanoscale, 11, 15448-15463. https://doi.org/10.1039/C9NR04065J

[8] Wang, X., Zeng, J., Yu, X., et al. (2020) Beetle-Like Droplet-Jumping Superamphiphobic Coatings for Enhancing Fog Collection of Sheet Arrays. RSC Advances, 10, 282-288. https://doi.org/10.1039/C9RA09329J

[9] Zheng, Z.K., Nottbohm, C.T., Turchanin, A., et al. (2010) Janus Nanomembranes: A Generic Platform for Chemistry in Two Dimensions. Angewandte Chemie International Edition, 49, 8493-8497. https://doi.org/10.1002/anie.201004053

[10] Ng, S.W., Noor, N. and Zheng, Z.J. (2018) Graphene-Based Two-Dimensional Janus Materials. NPG Asia Materials, 10, 217-237. 
https://doi.org/10.1038/s41427-018-0023-8

[11] Wang, Z.C., Li, Y.G., Li, S.H., et al. (2018) Janus Porous Membrane with Conical Nanoneedle Channel for Rapid Unidirectional Water Transport. Chemical Communications, 54, 10954-10957. https://doi.org/10.1039/C8CC05642K

[12] Yang, J., Li, H.-N., Chen, Z.-X., et al. (2019) Janus Membranes with Controllable Asymmetric Configurations for Highly Efficient Separation of Oil-in-Water Emulsions. Journal of Materials Chemistry A, 7, 7907-7917. https://doi.org/10.1039/C9TA00575G

[13] Zhang, Q., Li, Y., Yan, Y., et al. (2020) Highly Flexible Monolayered Porous Membrane with Superhydrophilicity-Hydrophilicity for Unidirectional Liquid Penetration. ACS Nano, 14, 7287-7296. https://doi.org/10.1021/acsnano.0c02558

[14] Shi, Y.L., Li, Y., Wu, J.M., et al. (2014) A Novel Transdermal Drug Delivery System Based on Self-Adhesive Janus Nanofibrous Film with High Breathability and Monodirectional Water-Penetration. Journal of Biomaterials Science, Polymer Edition, 25, 713-728. https://doi.org/10.1080/09205063.2014.897596

[15] Hou, L., Wang, N., Man, X., et al. (2019) Interpenetrating Janus Membrane for High Rectification Ratio Liquid Unidirectional Penetration. ACS Nano, 13, 4124-4132. https://doi.org/10.1021/acsnano.8b08753

[16] Shi, L., Liu, X., Wang, W., et al. (2019) A Self-Pumping Dressing for Draining Excessive Biofluid around Wounds. Advanced Materials, 31, Article ID: 1804187. https://doi.org/10.1002/adma.201804187

[17] Hou, L.L., Wang, N., Wu, J., et al. (2018) Bioinspired Superwettability Electrospun Micro/Nanofibers and Their Applications. Advanced Functional Materials, 28, Article ID: 1801114. https://doi.org/10.1002/adfm.201801114

[18] Doshi, J. and Reneker, D.H. (1995) Electrospinning Process and Applications of Electrospun Fibers. Journal of Electrostatics, 35, 151-160. https://doi.org/10.1016/0304-3886(95)00041-8

[19] Wu, J.D., Ding, Y.J., Wang, J.Q., et al. (2018) Facile Fabrication of Nanofiber- and Micro/Nanosphere-Coordinated PVDF Membrane with Ultrahigh Permeability of Viscous Water-in-Oil Emulsions. Journal of Materials Chemistry A, 6, 7014-7020. https://doi.org/10.1039/C8TA01539B

[20] Geng, Y.T., Zhang, P., Wang, Q.T., et al. (2017) Novel PAN/PVP Janus Ultrafine Fiber Membrane and Its Application for Biphasic Drug Release. Journal of Materials Chemistry B, 5, 5390-5396. https://doi.org/10.1039/C7TB00929A

[21] Yan, L., Yang, X., Long, J., et al. (2020) Universal Unilateral Electro-Spinning/Spraying Strategy to Construct Water-Unidirectional Janus Membranes with Well-Tuned Hierarchical Micro/Nanostructures. Chemical Communications, 56, 478-481. https://doi.org/10.1039/C9CC08088K

[22] Wang, H.X., Ding, J., Dai, L.M., et al. (2010) Directional Water-Transfer through Fabrics Induced by Asymmetric Wettability. Journal of Materials Chemistry, 20, 7938-7940. https://doi.org/10.1039/c0jm02364g 